\title{
Supersymmetric KdV equation: Darboux transformation and discrete systems
}

\author{
Ling-Ling Xue ${ }^{1}$, D. Levi ${ }^{2}$ and Q. P. Liu ${ }^{1}$ \\ ${ }^{1}$ Department of Mathematics, China University of Mining and Technology, Beijing \\ 100083, P. R. China \\ 2 Dipartimento di Matematica e Fisica, Universita' degli Studi di Roma Tre and \\ Sezione INFN Roma Tre, Via della Vasca Navale 84, I00146 Roma Italy \\ E-mail: qpl@cumtb.edu.cn
}

\begin{abstract}
For the supersymmetric KdV equation, a proper Darboux transformation is presented. This Darboux transformation leads to the Bäcklund transformation found early by Liu and Xie [1. The Darboux transformation and the related Bäcklund transformation are used to construct integrable super differential-difference and difference-difference systems. The continuum limits of these discrete systems and of their Lax pairs are also considered.
\end{abstract}

PACS numbers: 02.30.Ik, 02.70.Bf

Submitted to: J. Phys. A: Math. Gen. 


\section{Introduction}

In a seminal paper [2], Manin and Radul proposed a supersymmetric KP hierarchy. This integrable hierarchy was later studied intensively in [3, 4]. As in the classical case, the reductions to a finite number of fields of the supersymmetric KP hierarchy have been studied. The simplest and most important reduction is the supersymmetric KdV equation, which reads

$$
\alpha_{t}=\alpha_{x x x}+3(\alpha \mathcal{D} \alpha)_{x}
$$

where $\alpha=\alpha(t, x, \theta)$ is a fermionic super field depending on a temporal variable $t$ and the spatial super variables $(x, \theta)$ and $\mathcal{D}=\partial_{\theta}+\theta \partial_{x}$ is the corresponding super space derivative. $\theta$ is a fermionic commuting variable while $x$ is a bosonic anticommuting variable. Thus the fermionic (odd) variable $\theta$ is nilpotent and we can carry out the Taylor expansion of the fermonic super field $\alpha$ :

$$
\alpha=\rho(x, t)+\theta v(x, t)
$$

where the component fields $\rho(x, t)$ and $v(x, t)$ are fermionic and bosonic quantities respectively. In this way, the supersymmetric KdV equation (1) may be decomposed in the following system

$$
\begin{aligned}
& v_{t}=v_{x x x}+6 v v_{x}-3 \rho \rho_{x x}, \\
& \rho_{t}=\rho_{x x x}+3(v \rho)_{x} .
\end{aligned}
$$

This system may be compared with the super KdV proposed by Kupershmidt 34

$$
\begin{aligned}
& v_{t}=-v_{x x x}+6 v v_{x}-3 \rho \rho_{x x}, \\
& \rho_{t}=-4 \rho_{x x x}+6 v \rho_{x}+3 v_{x} \rho .
\end{aligned}
$$

As observed by Mathieu [8], even though the above two systems (2) and (3) appear similar, they are very different. In fact, only (2) is invariant under

$$
\tilde{v}=\varepsilon \rho_{x}, \tilde{\rho}=\varepsilon v,
$$

where $\varepsilon$ is a fermionic parameter. Thus (2) is known as the supersymmetric KdV equation while (3) is the fermionic $\mathrm{KdV}$ equation. More details on this may be found in [8] (see also [34]).

We can also write (1) in potential form, i.e.

$$
\beta_{t}=\beta_{x x x}+3 \beta_{x} \mathcal{D} \beta_{x}, \quad \alpha=\beta_{x} .
$$

Like the $\mathrm{KdV}$ equation, the supersymmetric $\mathrm{KdV}$ equation has many interesting properties; it is a bi-Hamiltonian system [5, 6], has a prolongation structure [7] and a Bäcklund transformation [1, possesses an infinite number of conserved quantities and satisfies the Painlevé property [ㅇ, 9], can be studied in the framework of Hirota bilinear method [10, 11, 12, etc. (see also [13, 14, 15, 16] for the most recent developments).

The existence of Darboux Transformations (DTs) is important for integrable systems (see, for example, the monographs [17, 18, 19] and the recent review article [20]). In general, one can introduce two kinds of Darboux transformations, the elementary 
DT and the binary DT, and from them we get Bäcklund transformations. For the supersymmetric KdV equation (11), the construction of DTs was initiated in [21] and continued in [22] and both elementary DT and binary DT have been constructed. Moreover, with the use of a super Gardner transformation, one has derived a Bäcklund transformation for the supersymmetric KdV equation [1], which reads

$$
\left(\beta_{[1]}+\beta\right)_{x}-2 p_{1}\left(\beta_{[1]}-\beta\right)+\frac{1}{2}\left(\beta_{[1]}-\beta\right) \mathcal{D}\left(\beta_{[1]}-\beta\right)=0
$$

where $p_{1}$ is the Bäcklund parameter. Moreover, (5) yields a superposition formula which may be applied to construct multi-soliton solutions.

Now it seems natural to ask ourselves which DT provides the Bäcklund transformation (5)? A careful consideration shows that none of DTs constructed in [21, 22] gives the Bäcklund transformation (5). Thus, a proper DT is missing for the supersymmetric KdV equation and one of the purposes of this paper is to fill this gap.

Apart from their importance in the construction of solutions, Darboux and Bäcklund transformations are known to play a key role for constructing nonlinear integrable discrete equations. Indeed, as showed by Levi and Benguria [23], Darboux and Bäcklund transformations can be used to provide integrable discretizations of the continuous nonlinear integrable systems (see also [24, 25, 26, 27]). Most recently, Grahovski and Mikhailov found integrable discretizations for a class of nonlinear Schrödinger equations on Grassmann algebras [28]. A generalization of this approach to supersymmetric integrable systems is up to now lacking and another purpose of this paper is to fill this gap. We will apply this idea to the supersymmetric $\mathrm{KdV}$ equation and show that the proper Darboux transformation enables us to discretize it and to obtain an integrable super differential-difference system and an integrable super difference-difference system.

The paper is organized as follows. In Section 2 we present a proper Darboux transformation for the supersymmetric $\mathrm{KdV}$ equation and derive its well known Bäcklund transformation. Then in Section 3 we use the obtained transformations to construct discrete integrable super systems. Both differential-difference equations and difference-difference equations are obtained. In Section 4, by performing various continuum limits, we show that our discrete systems are the proper discretizations of the potential supersymmetric KdV equation. The final Section summarizes the results and indicates the further directions of research. We leave to an Appendix the proof that the obtained nonlinear partial difference equation satisfies the compatibility around the cube but does not have the tetrahedron property.

\section{Darboux transformations for the supersymmetric $\mathrm{KdV}$}

Our result is summarized by the following Proposition.

Proposition 1 Let us suppose that the super field $\phi(x, \lambda)$ satisfies the linear supersymmetric equation

$$
\phi_{x x}+\alpha \mathcal{D} \phi=\lambda^{2} \phi
$$


and $\phi_{[0]}\left(x, p_{1}\right)$ be a solution of (마) for $\lambda=p_{1}$. Let us define

$$
\begin{aligned}
\phi_{[1]} & \equiv\left(\partial_{x}-\Lambda \mathcal{D}+p_{1}\right) \phi, \\
\alpha_{[1]} & \equiv \alpha+2 \Lambda_{x}, \quad \Lambda \equiv \frac{\phi_{[0], x}+p_{1} \phi_{[0]}}{\mathcal{D} \phi_{[0]}} .
\end{aligned}
$$

Then $\phi_{[1]}(x, \lambda)$ will satisfy the following linear supersymmetric equation

$$
\phi_{[1]_{x x}}+\alpha_{[1]}\left(\mathcal{D} \phi_{[1]}\right)=\lambda^{2} \phi_{[1]} .
$$

Proof. It is just a trivial direct calculation in which (7, 8) is introduced in (9) and $\phi$ it is shown to satisfy (6) .

The above DT (7, 8) may be regarded as a nontrivial extension of the DT given in [21]. Indeed, in Proposition 2.1 of [21] one has considered a solution of (6) with zero energy (namely $p_{1}=0$ ); here our seed solution $\phi_{[0]}$ is an (odd) solution of ([6) for an arbitrary value of $p_{1}$.

We show now that the DT (17, 8) leads to the Bäcklund transformation (5) derived by other techniques in [1]. In fact, let us introduce the potential $\beta_{[1]}$ such that $\alpha_{[1]}=\beta_{[1]_{x}}$, then the first equation in (8) gives

$$
\Lambda=\frac{1}{2}\left(\beta_{[1]}-\beta\right) \text {, }
$$

where the constant of integration is set to zero. On the other hand, taking into account that

$$
\phi_{[0], x}=\Lambda\left(\mathcal{D} \phi_{[0]}\right)-p_{1} \phi_{[0]}
$$

and

$$
\phi_{[0], x x}=p_{1}^{2} \phi_{[0]}-\alpha\left(\mathcal{D} \phi_{[0]}\right)
$$

we obtain

$$
\Lambda_{x}+\beta_{x}-2 p_{1} \Lambda+\Lambda(\mathcal{D} \Lambda)=0
$$

i.e. the Bäcklund transformation (5) for the supersymmetric $\mathrm{KdV}$ (1).

\section{Discrete equations from the super symmetric DT}

Let us rewrite the Darboux transformation (7, 8) in matrix form. Introducing the vector $\Psi=\left(\phi, \phi_{x}, \mathcal{D} \phi, \mathcal{D} \phi_{x}\right)^{\mathrm{T}}$, with the help of (15) one may rewrite (6) and (7) as the following systems

$$
\begin{aligned}
& \Psi_{x}=L \Psi, \quad L=\left(\begin{array}{cccc}
0 & 1 & 0 & 0 \\
\lambda^{2} & 0 & -\beta_{x} & 0 \\
0 & 0 & 0 & 1 \\
0 & \beta_{x} & \lambda^{2}-\mathcal{D} \beta_{x} & 0
\end{array}\right), \\
& \Psi_{[1]}=W \Psi, \quad W=\left(\begin{array}{cccc}
p_{1} & 1 & -\Lambda & 0 \\
\lambda^{2} & p_{1} & \left(\mathcal{D} \Lambda-2 p_{1}\right) \Lambda & -\Lambda \\
0 & \Lambda & p_{1}-\mathcal{D} \Lambda & 1 \\
\lambda^{2} \Lambda & \left(2 p_{1}-\mathcal{D} \Lambda\right) \Lambda & \lambda^{2}-2 p_{1}(\mathcal{D} \Lambda)+(\mathcal{D} \Lambda)^{2} & p_{1}-\mathcal{D} \Lambda
\end{array}\right),
\end{aligned}
$$


where $\Lambda$ is given by (10). The compatibility of the two linear systems (11, 12) is

$$
W_{x}+W L-L_{[1]} W=0,
$$

equivalent to the Bäcklund transformation (5). It is worthwhile to notice here that the matrix $W$ is a function, through $\Lambda$, of both $\beta$ and $\beta_{[1]}$. We can interpret (13) as a differential-difference equation by the following identifications

$$
\beta \equiv \beta_{n}(x), \quad \beta_{[1]} \equiv \beta_{n+1}(x) .
$$

(13) will admit as a Lax pair (11) and (12), therefore it will be integrable, at least in the Lax sense.

Let us introduce a new Darboux transformation

$$
\Psi_{[2]}=V \Psi,
$$

where the matrix $V$ is equal to the matrix $W$ with $p_{1}$ and $\beta_{[1]}$ replaced by $p_{2}$ and $\beta_{[2]}$ respectively, i.e. the matrix $V$ is a function through $\Lambda$ of both $\beta$ and $\beta_{[2]}$. If we consider the compatibility of (12) with (14), i.e. the Bianchi permutability of a Bäcklund transformation (13) of parameter $p_{1}$ with one of parameter $p_{2},\left(\Psi_{[1]}\right)_{[2]}=\left(\Psi_{[2]}\right)_{[1]}$, with $\beta_{[12]}=\beta_{[21]}$, we get that the following consistency condition

$$
W_{[2]} V=V_{[1]} W
$$

must be true. (15) leads to

$$
\beta_{[12]}=\beta+\frac{2\left(p_{1}+p_{2}\right)\left(\beta_{[1]}-\beta_{[2]}\right)}{2\left(p_{2}-p_{1}\right)+\mathcal{D}\left(\beta_{[1]}-\beta_{[2]}\right)}
$$

and

$$
\left(\beta_{[1]}-\beta_{[2]}\right)\left[2\left(\beta_{[1]}-\beta_{[2]}\right)_{x}+\left(\beta_{[1]}-\beta\right)\left(4\left(p_{2}-p_{1}\right)+\mathcal{D}\left(\beta_{[1]}-\beta_{[2]}\right)\right)\right]=0 .
$$

(16) is the superposition formula considered in [1] while (17) is an extra constraint.

To obtain a partial difference system, we use the following identifications

$$
\beta \equiv \beta_{n, m}(x), \quad \beta_{[1]} \equiv \beta_{n+1, m}(x), \quad \beta_{[2]} \equiv \beta_{n, m+1}(x), \quad \beta_{[12]} \equiv \beta_{n+1, m+1}(x),
$$

thus the system (16, 17) can be interpreted as an integrable differential-partial difference system as it contains the superderivative $\mathcal{D}$.

To find a differential-difference system, we rewrite the super fields in terms of its components. Let us assume that

$$
\beta=\xi+\theta u, \quad \phi=\varphi+\theta f,
$$

and define

$$
\xi \equiv \xi_{n}(x), \quad \xi_{[1]} \equiv \xi_{n+1}(x), \quad u \equiv u_{n}(x), \quad u_{[1]} \equiv u_{n+1}(x) .
$$

Then the Bäcklund transformation (13) is split into

$$
\begin{aligned}
& \left(\xi_{n+1}+\xi_{n}\right)_{x}=2 p_{1}\left(\xi_{n+1}-\xi_{n}\right)-\frac{1}{2}\left(u_{n+1}-u_{n}\right)\left(\xi_{n+1}-\xi_{n}\right) \\
& \left(u_{n+1}+u_{n}\right)_{x}=2 p_{1}\left(u_{n+1}-u_{n}\right)-\frac{1}{2}\left(u_{n+1}-u_{n}\right)^{2}-\left(\xi_{n+1}-\xi_{n}\right)\left(\xi_{n}\right)_{x}
\end{aligned}
$$


which is a differential-difference system.

Introducing $\chi_{n}=\left(\left(\varphi_{n}\right),\left(\varphi_{n}\right)_{x},\left(f_{n}\right),\left(f_{n}\right)_{x}\right)^{\mathrm{T}}$, it follows from (6), (17) and (18) that

$\left(\chi_{n}\right)_{x}=\mathcal{L}_{n} \chi_{n}, \quad \mathcal{L}_{n}=\left(\begin{array}{cccc}0 & 1 & 0 & 0 \\ \lambda^{2} & 0 & -\left(\xi_{n}\right)_{x} & 0 \\ 0 & 0 & 0 & 1 \\ 0 & \left(\xi_{n}\right)_{x} & \lambda^{2}-\left(u_{n}\right)_{x} & 0\end{array}\right)$

and

$\chi_{n+1}=\mathcal{W}_{n} \chi_{n}, \quad \mathcal{W}_{n}=\left(\begin{array}{cccc}p_{1} & 1 & -\eta & 0 \\ \lambda^{2} & p_{1} & -2 p_{1} \eta+\eta g & -\eta \\ 0 & \eta & p_{1}-g & 1 \\ \lambda^{2} \eta & 2 p_{1} \eta-\eta g & \lambda^{2}-2 p_{1} g+g^{2} & p_{1}-g\end{array}\right)$

with

$$
\eta \equiv \frac{1}{2}\left(\xi_{n+1}-\xi_{n}\right), \quad g \equiv \frac{1}{2}\left(u_{n+1}-u_{n}\right) .
$$

Then the compatibility condition of (19) and (20) gives

$$
\left(\mathcal{W}_{n}\right)_{x}+\mathcal{W}_{n} \mathcal{L}_{n}-\mathcal{L}_{n+1} \mathcal{W}_{n}=0
$$

which holds if and only if (18) is satisfied. In the next Section, we will show that, after a continuum limit, the system (18) leads to the potential supersymmetric KdV equation, thus it constitutes a discretization of the potential supersymmetric KdV equation.

To find a difference-difference system, we define

$$
\begin{aligned}
& \xi \equiv \xi_{n, m}, \quad \xi_{[1]} \equiv \xi_{n+1, m}, \quad \xi_{[2]} \equiv \xi_{n, m+1}, \quad \xi_{[12]} \equiv \xi_{n+1, m+1}, \\
& u \equiv u_{n, m}, \quad u_{[1]} \equiv u_{n+1, m}, \quad u_{[2]} \equiv u_{n, m+1}, \quad u_{[12]} \equiv u_{n+1, m+1} \text {. }
\end{aligned}
$$

As above, we consider another Darboux transformation, equivalent to (14), which introducing $\chi_{n, m}=\left(\left(\varphi_{n, m}\right),\left(\varphi_{n, m}\right)_{x},\left(f_{n, m}\right),\left(f_{n, m}\right)_{x}\right)^{\mathrm{T}}$, now reads

$$
\chi_{n, m+1}=\mathcal{V}_{n, m} \chi_{n, m},
$$

where the matrix $\mathcal{V}_{n, m}$ is the matrix $\mathcal{W}_{n}=\mathcal{W}_{n, m}$ of (20) with $p_{1}, \xi_{n+1}=\xi_{n+1, m}$ and $u_{n+1}=u_{n+1, m}$ replaced by $p_{2}, \xi_{n, m+1}$ and $u_{n, m+1}$ respectively.

Now the compatibility condition of (20), written as $\chi_{n+1, m}=\mathcal{W}_{n, m} \chi_{n, m}$, and (21), namely

$$
\mathcal{W}_{n, m+1} \mathcal{V}_{n, m}=\mathcal{V}_{n+1, m} \mathcal{W}_{n, m}
$$

yields an integrable difference-difference system

$$
\begin{aligned}
& \xi_{n+1, m+1}=\xi_{n, m}+\frac{2\left(p_{1}+p_{2}\right)\left(\xi_{n+1, m}-\xi_{n, m+1}\right)}{2\left(p_{2}-p_{1}\right)+u_{n+1, m}-u_{n, m+1}}, \\
& u_{n+1, m+1}=u_{n, m}+\frac{2\left(p_{1}+p_{2}\right)\left(u_{n+1, m}-u_{n, m+1}\right)}{2\left(p_{2}-p_{1}\right)+u_{n+1, m}-u_{n, m+1}} \\
& \quad-\frac{\left(p_{1}+p_{2}\right)\left(4\left(p_{2}-p_{1}\right)+u_{n+1, m}-u_{n, m+1}\right)}{\left(2\left(p_{2}-p_{1}\right)+u_{n+1, m}-u_{n, m+1}\right)^{2}}\left(\xi_{n+1, m}-\xi_{n, m+1}\right)\left(\xi_{n, m+1}-\xi_{n, m}\right) .
\end{aligned}
$$


In the next Section, we will show that, after a double continuum limit, the system (22) leads to the potential supersymmetric KdV equation, thus it constitutes a discretization of the potential supersymmetric KdV equation.

\section{Remarks:}

(i) The difference-difference system (22) may also be derived from (16) and (17) directly. Indeed, one can write (16) in components and obtain (22a) and

$$
\begin{aligned}
u_{n+1, m+1} & =u_{n, m}+\frac{2\left(p_{1}+p_{2}\right)\left(u_{n+1, m}-u_{n, m+1}\right)}{2\left(p_{2}-p_{1}\right)+u_{n+1, m}-u_{n, m+1}} \\
& +\frac{2\left(p_{1}+p_{2}\right)\left(\xi_{n+1, m}-\xi_{n, m+1}\right)\left(\xi_{n+1, m}-\xi_{n, m+1}\right)_{x}}{\left(2\left(p_{2}-p_{1}\right)+u_{n+1, m}-u_{n, m+1}\right)^{2}} .
\end{aligned}
$$

(17) gives

$$
\begin{aligned}
& 2\left(\xi_{n+1, m}-\xi_{n, m+1}\right)\left(\xi_{n+1, m}-\xi_{n, m+1}\right)_{x} \\
& =-\left(\xi_{n+1, m}-\xi_{n, m+1}\right)\left(\xi_{n, m+1}-\xi_{n, m}\right)\left(4\left(p_{2}-p_{1}\right)+u_{n+1, m}-u_{n, m+1}\right),
\end{aligned}
$$

from which we can eliminate the differential part of (23) and obtain (22b).

(ii) If $\xi=0,(22)$ reduces to the well-known lattice potential $\mathrm{KdV}$ equation

$$
u_{n+1, m+1}=u_{n, m}+\frac{2\left(p_{1}+p_{2}\right)\left(u_{n+1, m}-u_{n, m+1}\right)}{2\left(p_{2}-p_{1}\right)+u_{n+1, m}-u_{n, m+1}} .
$$

(iii) The system (22) is integrable in the sense that it possesses Lax representation. In the appendix, it will be shown that this system is consistent around a cube, i.e. it has the CAC property [29].

\section{Continuum limits of the discrete equations obtained in the previous Section}

We can characterize the discrete systems obtained in the previous Section by analyzing their continuum limits [30, 31]. We obtained the differential-difference system (18) and

the difference-difference system (22) as discrete versions of the potential supersymmetric $\mathrm{KdV}$ equation. We now justify this claim by considering their continuum limits.

\subsection{The continuous limit of (18)}

To carry out the continuous limit of (18) we define the new continuous variable $\tau$ as

$$
\xi_{n}(x) \equiv \xi(x, \tau), \quad u_{n}(x) \equiv u(x, \tau), \quad \tau=\frac{n}{p_{1}} .
$$

Then

$$
\xi_{n+1}(x) \equiv \xi\left(x, \tau+\frac{1}{p_{1}}\right), \quad u_{n+1}(x) \equiv u\left(x, \tau+\frac{1}{p_{1}}\right)
$$

can be expanded in $\frac{1}{p_{1}}$, and defining a new independent variable $t$ in term of $\tau$ and $x$ such that

$$
\partial_{\tau}=\partial_{x}+\frac{1}{12 p_{1}^{2}} \partial_{t}
$$


we obtain in the continuous limit up to terms of order $\frac{1}{p_{1}}$

$$
\xi_{t}=\xi_{x x x}+3 \xi_{x} u_{x}, \quad u_{t}=u_{x x x}+3 u_{x}^{2}+3 \xi_{x x} \xi_{x}
$$

which is the potential form of supersymmetric $\mathrm{KdV}$ equation (41).

\subsection{The semi-continuous limits of (22)}

We present here two different results obtained by implementing different continuous limits of (22), at first when we send to infinity just one of the discrete variables and secondly when we send to infinity a combination of both discrete variables.

4.2.1. Straight continuum limit The system (22) may be regarded as a discrete analogue of the differential-difference system (18). To see it, let us define

$$
\xi_{n, m} \equiv \xi_{n}(x), \quad u_{n, m} \equiv u_{n}(x), \quad x=\frac{m}{p_{2}} .
$$

For $\frac{1}{p_{2}}$ small, we have the following Taylor series expansions

$$
\begin{aligned}
& \xi_{k, m+1}=\xi_{k}\left(x+\frac{1}{p_{2}}\right)=\xi_{k}+\frac{1}{p_{2}} \xi_{k, x}+O\left(\frac{1}{p_{2}^{2}}\right), \\
& u_{k, m+1}=u_{k}\left(x+\frac{1}{p_{2}}\right)=u_{k}+\frac{1}{p_{2}} u_{k, x}+O\left(\frac{1}{p_{2}^{2}}\right),
\end{aligned}
$$

where $k$ can take the value $n$ or $n+1$. Substituting the above expansions into (22), the leading terms yield

$$
\begin{aligned}
& \left(\xi_{n+1}+\xi_{n}\right)_{x}=2 p_{1}\left(\xi_{n+1}-\xi_{n}\right)-\frac{1}{2}\left(\xi_{n+1}-\xi_{n}\right)\left(u_{n+1}-u_{n}\right) \\
& \left(u_{n+1}+u_{n}\right)_{x}=2 p_{1}\left(u_{n+1}-u_{n}\right)-\frac{1}{2}\left(u_{n+1}-u_{n}\right)^{2}-\left(\xi_{n+1}-\xi_{n}\right) \xi_{n, x}
\end{aligned}
$$

i.e. (18).

4.2.2. Skew continuum limit In this case, we introduce the new discrete variable

$$
N=n+m, \quad p_{2}=p_{1}+\epsilon, \quad \tau=\epsilon m,
$$

and

$$
\begin{aligned}
\xi_{n, m} & \equiv \xi_{N}(\tau), & u_{n, m} & \equiv u_{N}(\tau), \\
\xi_{n+1, m} & \equiv \xi_{N+1}(\tau), & u_{n+1, m} & \equiv u_{N+1}(\tau), \\
\xi_{n, m+1} & \equiv \xi_{N+1}(\tau+\epsilon), & u_{n, m+1} & \equiv u_{N+1}(\tau+\epsilon), \\
\xi_{n+1, m+1} & \equiv \xi_{N+2}(\tau+\epsilon), & u_{n+1, m+1} & \equiv u_{N+2}(\tau+\epsilon),
\end{aligned}
$$

and develop all dependent variables in Taylor series in $\epsilon$. After inserting them into (22), the leading order terms give

$$
\begin{aligned}
& \xi_{N+1, \tau}=-\frac{1}{4 p_{1}}\left(\xi_{N+2}-\xi_{N}\right)\left(2-u_{N+1, \tau}\right), \\
& \left(u_{N+2}-u_{N}\right)\left(2-u_{N+1, \tau}\right)^{2}+4 p_{1} u_{N+1, \tau}\left(2-u_{N+1, \tau}\right)=2 p_{1} \xi_{N+1, \tau}\left(4-u_{N+1, \tau}\right)\left(\xi_{N+1}-\xi_{N}\right) .
\end{aligned}
$$


Solving this system with respect to $\xi_{N+1, \tau}$ and $u_{N+1, \tau}$, and shifting $N+1$ to $N$, we get, either

$\xi_{N, \tau}=\frac{2\left(\xi_{N+1}-\xi_{N-1}\right)}{u_{N+1}-u_{N-1}-4 p_{1}}$

$u_{N, \tau}=\frac{2\left(u_{N+1}-u_{N-1}\right)}{u_{N+1}-u_{N-1}-4 p_{1}}+\frac{\left(u_{N+1}-u_{N-1}-8 p_{1}\right)}{\left(u_{N+1}-u_{N-1}-4 p_{1}\right)^{2}}\left(\xi_{N+1}-\xi_{N-1}\right)\left(\xi_{N}-\xi_{N-1}\right)$,

or the trivial solution

$$
\xi_{N, \tau}=0, \quad u_{N, \tau}=2 .
$$

If the fermionic variable $\xi_{N}$ is null, the system (28) is reduced to

$$
u_{N, \tau}=\frac{2\left(u_{N+1}-u_{N-1}\right)}{u_{N+1}-u_{N-1}-4 p_{1}},
$$

a differential-difference equation related to the Kac-van Moerbeke equation [32]. Thus, with this limit, the system (28) is a super extension of the Kac-van Moerbeke equation.

\subsection{Full continuum limit}

Two different semi-continuum limits have been considered for (22) and two differentialdifference systems, namely (25) and (28), are obtained. As (25) is equal to (18) its continuous limit is contained in Section (4.1) and it leads to the potential supersymmetric KdV equation (4). In the following we will consider the continuum limit of (28) and it will turn out that it also leads to the potential supersymmetric KdV equation (4).

For (28), defining

$$
\xi_{N}(\tau) \equiv \xi(s, \tau), \quad u_{N}(\tau) \equiv u(s, \tau), \quad s=\frac{N}{p_{1}},
$$

expanding

$$
\xi_{N \pm 1}(\tau) \equiv \xi\left(s \pm \frac{1}{p_{1}}, \tau\right), \quad u_{N \pm 1}(\tau) \equiv u\left(s \pm \frac{1}{p_{1}}, \tau\right)
$$

in $\frac{1}{p_{1}}$, and redefining the independent variables from $s, \tau$ to $x, t$

$$
\partial_{s}=\partial_{x}, \quad \partial_{\tau}=-\frac{1}{p_{1}^{2}} \partial_{x}-\frac{1}{6 p_{1}^{4}} \partial_{t}
$$

we get once again (24).

\subsection{Semi-continuous limits of the Lax pair}

The continuum limits considered above were done on the level of the nonlinear systems. We actually can work out also the continuum limits of the corresponding Lax pair (20) and (21):

$$
\left(p_{1}-\lambda\right) \chi_{n+1, m}=\mathcal{W}_{n, m} \chi_{n, m}, \quad\left(p_{2}-\lambda\right) \chi_{n, m+1}=\mathcal{V}_{n, m} \chi_{n, m}
$$


in which the matrices $\mathcal{W}$ and $\mathcal{V}$ are defined in Section 3. We now perform at first the skew continuum limit (26) on the Lax pair in order to obtain a Lax pair for (28). Using (26, 27) the first element of the Lax pair (29) reads

$\left(p_{1}-\lambda\right) \chi_{N+1}=\mathcal{W}_{N} \chi_{N}, \quad \mathcal{W}_{N}=\left(\begin{array}{cccc}p_{1} & 1 & -\zeta & 0 \\ \lambda^{2} & p_{1} & \left(h-2 p_{1}\right) \zeta & -\zeta \\ 0 & \zeta & p_{1}-h & 1 \\ \lambda^{2} \zeta & \left(2 p_{1}-h\right) \zeta & \lambda^{2}+\left(h-2 p_{1}\right) h & p_{1}-h\end{array}\right)$,

where $\zeta \equiv \frac{1}{2}\left(\xi_{N+1}-\xi_{N}\right), h \equiv \frac{1}{2}\left(u_{N+1}-u_{N}\right)$. Expanding the second element of the Lax pair (29) in $\epsilon$, we obtain

$$
\left(\epsilon+p_{1}-\lambda\right)\left(\chi_{N+1}+\epsilon \chi_{N+1, \tau}+\cdots\right)=\mathcal{W}_{N} \chi_{N}+\epsilon \mathcal{T}_{N} \chi_{N}+\cdots
$$

where

$$
\mathcal{T}_{N}=\left(\begin{array}{cccc}
1 & 0 & -\frac{1}{2} \xi_{N+1, \tau} & 0 \\
0 & 1 & t_{23} & -\frac{1}{2} \xi_{N+1, \tau} \\
0 & \frac{1}{2} \xi_{N+1, \tau} & 1-\frac{1}{2} u_{N+1, \tau} & 0 \\
\frac{1}{2} \lambda^{2} \xi_{N+1, \tau} & -t_{23} & t_{43} & 1-\frac{1}{2} u_{N+1, \tau}
\end{array}\right)
$$

with

$$
\begin{aligned}
& t_{23} \equiv-p_{1} \xi_{N+1, \tau}-2 \zeta+\frac{1}{2}\left(h \xi_{N+1, \tau}+\zeta u_{N+1, \tau}\right), \\
& t_{43} \equiv-p_{1} u_{N+1, \tau}-2 h+h u_{N+1, \tau} .
\end{aligned}
$$

Next with the help of (30) we obtain as the coefficient of the leading term of order $O(\epsilon)$ the following equation

$$
\left(p_{1}-\lambda\right) \chi_{N+1, \tau}=\mathcal{T}_{N} \chi_{N}-\chi_{N+1}
$$

The consistency condition of (30) and (31)

$$
\mathcal{W}_{N, \tau} \mathcal{W}_{N-1}+\mathcal{W}_{N} \mathcal{T}_{N-1}-\mathcal{T}_{N} \mathcal{W}_{N-1}=0
$$

leads to (28), thus (30, 31) constitute a Lax pair for the supersymmetric Kac-van Moerbeke equation (28).

\section{Conclusion}

In this paper, we give a new Darboux transformation for the supersymmetric KdV equation. By means of this Darboux transformation, the supersymmetric KdV equation is discretized. Both differential-difference system and difference-difference system are obtained, their integrability is shown and various continuum limits are considered.

This work opens the way to the construction of many discrete supersymmetric equations. We are planning to apply the idea to other supersymmetric integrable equations. In particular we are considering the supersymmetric Schrödinger equation [33] and the Kupershimidt's super KdV equation [34], which is a fermionic rather than supersymmetric extension of the KdV equation. 


\section{Acknowledgments}

LLX and QPL are supported by the National Natural Science Foundation of China (grant numbers: 10971222, 11271366 and 11331008) and the Fundamental Research Funds for Central Universities. LD has been partly supported by the Italian Ministry of Education and Research, PRIN "Continuous and discrete nonlinear integrable evolutions: from water waves to symplectic maps" and thanks the China University of Mining and Technology for its support during his visit to Beijing where this work has been carried out.

\section{Appendix: CAC property of the system (22)}

An integrable partial difference equation defined on a square lattice is characterized by the Consistency-Around-the-Cube (CAC) property [29, 35, 36], equivalent to the Bianchi permutability theorem for Bäcklund transformations of integrable nonlinear partial differential equations. In the following we will show that also the system (22) possesses such a property.

From (22), by adding a third direction, we have the following equations

$\xi_{[i j]}=\xi+f_{[i j]}\left(\xi_{[i]}-\xi_{[j]}\right), \quad u_{[i j]}=u+f_{[i j]}\left(u_{[i]}-u_{[j]}\right)+g_{[i j]}\left(\xi_{[i]}-\xi_{[j]}\right)\left(\xi_{[j]}-\xi\right)$,

where

$$
f_{[i j]} \equiv \frac{2\left(p_{i}+p_{j}\right)}{2\left(p_{j}-p_{i}\right)+u_{[i]}-u_{[j]}}, \quad g_{[i j]} \equiv-\frac{\left(p_{i}+p_{j}\right)\left(4\left(p_{j}-p_{i}\right)+u_{[i]}-u_{[j]}\right)}{\left(2\left(p_{j}-p_{i}\right)+u_{[i]}-u_{[j]}\right)^{2}}
$$

with $1 \leq i, j \leq 3$. Then we obtain

$\xi_{[i j l]}=\xi_{[l]}+f_{[i j l]}\left(\xi_{[i l]}-\xi_{[j l]}\right), \quad u_{[i j l]}=u_{[l]}+f_{[i j l]}\left(u_{[i l]}-u_{[j l]}\right)+g_{[i j l]}\left(\xi_{[i l]}-\xi_{[j l]}\right)\left(\xi_{[j l]}-\xi_{[l]}\right)$, where

$f_{[i j l]} \equiv \frac{2\left(p_{i}+p_{j}\right)}{2\left(p_{j}-p_{i}\right)+u_{[i l]}-u_{[j l]}}, \quad g_{[i j l]} \equiv-\frac{\left(p_{i}+p_{j}\right)\left(4\left(p_{j}-p_{i}\right)+u_{[i l]}-u_{[j l]}\right)}{\left(2\left(p_{j}-p_{i}\right)+u_{[i l]}-u_{[j l]}\right)^{2}}$.

Consistency means that $\xi_{[123]}=\xi_{[231]}=\xi_{[312]}, u_{[123]}=u_{[231]}=u_{[312]}$. Indeed, by direct calculation we find

$$
\begin{aligned}
\xi_{[123]} & =\frac{1}{H} \sigma_{i j l} \xi_{[i]}\left(p_{i}+p_{j}\right)\left(p_{i}+p_{l}\right)\left(2\left(p_{j}-p_{l}\right)-u_{[j]}+u_{[l]}\right) \\
& +\frac{1}{2 H^{2}}\left(\xi_{[1]}-\xi_{[3]}\right)\left(\xi_{[2]}-\xi_{[3]}\right)\left(\xi_{[3]}-\xi\right)\left(p_{1}+p_{2}\right)\left(p_{1}+p_{3}\right)\left(p_{2}+p_{3}\right) \sigma_{i j l}\left(p_{i}-p_{j}\right) u_{[l]}, \\
u_{[123]} & =\frac{1}{H} \sigma_{i j l} p_{i}^{2}\left[u_{[i]}\left(u_{[l]}-u_{[j]}\right)+2 p_{j}\left(u_{[i]}-u_{[j]}+u_{[l]}\right)-2 p_{l}\left(u_{[i]}+u_{[j]}-u_{[l]}\right)\right] \\
& +\frac{1}{2 H^{2}}\left(p_{1}+p_{2}\right)\left(p_{1}+p_{3}\right)\left(p_{2}+p_{3}\right) \\
& \times\left\{\sigma_{i j l} \xi \xi_{[i]}\left(2\left(p_{l}-p_{j}\right)+u_{[j]}-u_{[l]}\right)\left[p_{i}\left(u_{[j]}-u_{[l]}\right)+p_{j}\left(u_{[l]}-u_{[i]}\right)+p_{l}\left(u_{[i]}-u_{[j]}\right)\right]\right. \\
& +\sigma_{i j l} \xi_{[j]} \xi_{[l]}\left[8\left(p_{i}-p_{j}\right)\left(p_{i}-p_{l}\right)\left(p_{j}-p_{l}\right)+\left(p_{j}-p_{l}\right)\left(u_{[i]}-u_{[j]}\right)\left(u_{[i]}-u_{[l]}\right)\right. \\
& \left.\left.-4 u_{[i]}\left(p_{j}-p_{l}\right)\left(p_{i}-p_{j}-p_{l}\right)-2 u_{[j]}\left(p_{i}-p_{l}\right)\left(p_{i}-p_{j}+2 p_{l}\right)+2 u_{[l]}\left(p_{i}-p_{j}\right)\left(p_{i}+2 p_{j}-p_{l}\right)\right]\right\},
\end{aligned}
$$


where

$$
H \equiv 2\left(p_{1}-p_{2}\right)\left(p_{1}-p_{3}\right)\left(p_{2}-p_{3}\right)+\sigma_{i j l}\left(p_{i}^{2}-p_{j}^{2}\right) u_{[l]},
$$

and $\sigma_{i j l}$ denotes the cyclic sum over the subscripts $(i, j, l)=(1,2,3),(2,3,1),(3,1,2)$. Then $\xi_{[123]}$ and $u_{[123]}$ are symmetric under any permutation of $(1,2,3)$, thus $(22)$ obeys the CAC property. We notice that since both $\xi_{[123]}$ and $u_{[123]}$ are independent of $u$ but dependent on $\xi$, the system (22) does not have the tetrahedron property.

\section{References}

[1] Liu Q P and Xie Y F 2004 Nonlinear superposition formula for N=1 supersymmetric KdV equation Phys. Lett. A 325 139-143

[2] Manin Yu and Radul A O 1985 A supersymmetric extension of the Kadomtsev-Petviashvili hierarchy Commun. Math. Phys. 98 65-77

[3] Mulase M 1991 A new super KP system and a characterization of the Jacobians of arbitrary algebraic super curves J. Diff. Geom. 34 651-680

[4] Ueno K and Yamada H 1988 Supersymmetric extension of the Kadomtsev-Petviashvili hierarchy and the universal super Grassmann manifold Adv. Stud. Pure Math. 16 373-426

[5] Figueroa-O'Farrill J M, Ramos E and Mas J 1991 Integrability and biHamiltionian structure of the even order sKdV hierarchies Rev. Math. Phys. 3 479-501

[6] Oevel W and Popowicz Z 1991 The bi-Hamiltonian structure of fully supersymmetric Korteweg-de Vries systems Commun. Math. Phys. 139 441-460

[7] Roelofs G H M and Hijligenberg van den N W 1990 Prolongation structures for supersymmetric equations J. Phys. A: Math. Gen. 23 5117-5130

[8] Mathieu P 1988 Supersymmetric extension of the Korteweg-de Vries equation J. Math. Phys. 29 2499-2506

[9] Mathieu P 1988 The Painlevé property for fermionic extensions of the Korteweg-de Vries equation Phys. Lett. A 128 169-171

[10] Carstea A S, Ramani A and Grammaticos B 2001 Constructing the soliton solutions for the $N=1$ supersymmetric KdV hierarchy Nonlinearity 14 1419-1424

[11] Liu Q P and Hu X B 2005 Bilinearization of $N=1$ supersymmetric Korteweg-de Vries equation revisited J. Phys. A: Math. Gen. 38 6371-6378

[12] McArthur I N and Yung C M 1993 Hirota bilinear form for the Super-KdV hierarchy Mod. Phys. Lett. A 8 1739-1745

[13] Fan E G and Hon Y C 2012 Super extension of Bell polynomials with applications to supersymmetric equations J. Math. Phys. 53013503

[14] Gao X N and Lou S Y 2012 Bosonization of supersymmetric KdV equation Phys. Lett. B 707 $209-215$

[15] Gao X N, Lou S Y and Tang X Y 2013 Bosonization, singularity analysis, nonlocal symmetry reductions and exact solutions of supersymmetric KdV equation J. High Energy Phys. JHEP029

[16] Hon Y C and Fan E G 2011 Super quasi-periodic wave solutions and asymptotic analysis for $N=1$ supersymmetric KdV-type equations Theor. Math. Phys. 166 317-336

[17] Doktorov E V and Leble S B 2007 A dressing method in mathematical physics (Berlin: SpringerVerlag)

[18] Gu C H, Hu H S and Zhou Z X 2005 Darboux transformations in integrable systems: theory and their applications to geometry (Berlin: Springer-Verlag)

[19] Matveev V B and Salle M A 1991 Darboux transformations and solitons (Berlin: Springer-Verlag)

[20] Cieśliński J L 2009 Algebraic construction of the Darboux matrix revisited J. Phys. A: Math. Theor. 42404003 
[21] Liu Q P 1995 Darboux transformations for supersymmetric Korteweg-de Vries equations Lett. Math. Phys. 35 115-122

[22] Liu Q P and Mañas M 1998 Pfaffian solutions for the Manin-Radul-Mathieu SUSY KdV and SUSY sine-Gordon equations Phys. Lett. B 436 306-310

[23] Levi D and Benguria R 1980 Bäcklund transformations and nonlinear differential difference equations Proc. Natl. Acad. Sci. USA 77 5025-5027

[24] Levi D 1981 Nonlinear differential difference equations as Bäcklund transformations J. Phys. A: Math. Gen. 14 1083-1098

[25] Schief W K 1996 Self-dual Einstein spaces via a permutability theorem for the Tzitzeica equation Phys. Lett. A 223 55-62

[26] Nijhoff F W, Quispel G R W and Capel H W 1983 Direct linearization of nonlinear differencedifference equations Phys. Lett. A 97 125-128

[27] Quispel G R W, Nijhoff F W, Capel H W and van der Linden J 1984 Linear integral equations and nonlinear difference-difference equations Physica A 125 344-380

[28] Grahovski G G and Mikhailov A V Integrable discretisations for a class of nonlinear Schrödinger equations on Grassmann algebras arXiv:1303.1853, Phys. Lett. A (to appear)

[29] Adler V E, Bobenko A I and Suris Yu B 2003 Classification of integrable equations on quad-graphs. The consistency approach Commun. Math. Phys. 233 513-543

[30] Nijhoff F W and Capel H W 1995 The discrete Korteweg-de Vries equation Acta Appl. Math. 39 $133-158$

[31] Nijhoff F W Discrete systems and integrability Math3491/5491 lecture notes (University of Leeds)

[32] Kac M and van Moerbeke P 1975 On an explicitly soluble system on nonlinear differential equations related to certain Toda lattices Adv. Math. 16 160-169

[33] Roelofs G H M and Kersten P H M 1992 Supersymmetric extensions of the nonlinear Schrödinger equation: symmetries and coverings J. Math. Phys. 33 2185-2206

[34] Kupershmidt B A 1984 A super Korteweg-de Vries equation: an integrable system Phys. Lett. A $102213-215$

[35] Hietarinta J 2004 A new two-dimensional lattice model that is 'consistent around a cube' J. Phys. A: Math. Gen. 37 L67-L73

[36] Hietarinta J 2005 Searching for CAC-maps J. Nonlinear Math. Phys. 12 (Suppl. 2) 223-230 